\title{
On the existence of branched coverings between surfaces with prescribed branch data, I
}

\author{
EKATERINA PERVOVA \\ CARlo Petronio
}

For the existence of a branched covering $\widetilde{\Sigma} \rightarrow \Sigma$ between closed surfaces there are easy necessary conditions in terms of $\chi(\widetilde{\Sigma}), \chi(\Sigma)$, orientability, the total degree, and the local degrees at the branching points. A classical problem dating back to Hurwitz asks whether these conditions are also sufficient. Thanks to the work of many authors, the problem remains open only when $\Sigma$ is the sphere, in which case exceptions to existence are known to occur. In this paper we describe new infinite series of exceptions, in particular previously unknown exceptions with $\widetilde{\Sigma}$ not the sphere and with more than three branching points. All our series come with systematic explanations, based on several different techniques (including dessins d'enfants and decomposability) that we exploit to attack the problem, besides Hurwitz's classical technique based on permutations. Using decomposability we also establish an easy existence result.

57M12; 57M30, 57N05

\section{Problem and new partial solutions}

In this section we state the Hurwitz existence problem, we outline its relevance to other areas of topology and our motivations for picking it up, and we state our new contributions towards its solution, also explaining the techniques we have used to obtain them. We address the reader to Section 2 for an overview of the known results and techniques, which will help putting our results into context.

Basic definitions A branched covering is a map $f: \widetilde{\Sigma} \rightarrow \Sigma$, where $\widetilde{\Sigma}$ and $\Sigma$ are closed connected surfaces and $f$ is locally modelled on maps of the form $\mathbb{C} \ni z \mapsto z^{k} \in \mathbb{C}$ for some $k \geqslant 1$. The integer $k$ is called the local degree at the point of $\widetilde{\Sigma}$ corresponding to 0 in the source $\mathbb{C}$. If $k>1$ then the point of $\Sigma$ corresponding to 0 in the target $\mathbb{C}$ is called a branching point. The branching points are isolated, hence there are finitely

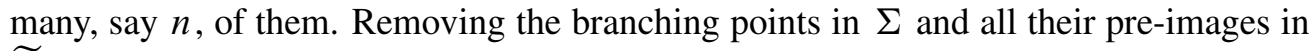
$\widetilde{\Sigma}$, the restriction of $f$ gives a genuine covering, whose degree we will denote by $d$. 
If the $i$-th branching point on $\Sigma$ has $m_{i}$ pre-images, the local degrees $\left(d_{i j}\right)_{j=1, \ldots, m_{i}}$ at these points give a partition of $d$, namely $d_{i j} \geqslant 1$ and $\sum_{j=1}^{m_{i}} d_{i j}=d$. In the sequel we will always assume that in a partition $\left(d_{1}, \ldots, d_{m}\right)$ of $d$ we have $d_{1} \geqslant \ldots \geqslant d_{m}$, which allows us to regard the partition as an array of integers rather than an unordered set with repetitions.

Branch data Suppose we are given closed connected surfaces $\widetilde{\Sigma}$ and $\Sigma$, integers $n \geqslant 0$ and $d \geqslant 2$, and for $i=1, \ldots, n$ a partition $\left(d_{i j}\right)_{j=1, \ldots, m_{i}}$ of $d$. The 5-tuple $\left(\widetilde{\Sigma}, \Sigma, n, d,\left(d_{i j}\right)\right)$ will be called the branch datum of a candidate branched covering. To such a datum we will always associate the integer $\tilde{n}$ defined as $m_{1}+\ldots+m_{n}$.

Compatibility We define a branch datum to be compatible if the following conditions hold:

(1) $\chi(\widetilde{\Sigma})-\tilde{n}=d \cdot(\chi(\Sigma)-n)$;

(2) $n \cdot d-\tilde{n}$ is even;

(3) If $\Sigma$ is orientable then $\widetilde{\Sigma}$ is also orientable;

(4) If $\Sigma$ is non-orientable and $d$ is odd then $\widetilde{\Sigma}$ is also non-orientable;

(5) If $\Sigma$ is non-orientable but $\widetilde{\Sigma}$ is orientable then each partition $\left(d_{i j}\right)_{j=1, \ldots, m_{i}}$ of $d$ refines the partition $(d / 2, d / 2)$.

The meaning of Condition (5) is that $\left(d_{i j}\right)_{j=1, \ldots, m_{i}}$ is obtained by juxtaposing two partitions of $d / 2$ and reordering. Note that $d$ is even by Condition (4).

The problem It is not too difficult to show that if a branched covering $\widetilde{\Sigma} \rightarrow \Sigma$ exists then the corresponding branch datum, with $n, d,\left(d_{i j}\right), \tilde{n}$ defined as above, is compatible. Conditions (1), (3), and (4) are obvious, Condition (5) follows from the fact that the covering factors through the orientation covering of $\Sigma$, and a short proof of Condition (2) will be given for the sake of completeness in Section 3.

We will call Hurwitz existence problem the question of which compatible branch data are actually realized by some branched covering. In the sequel we will always consider the branch datum $\left(\widetilde{\Sigma}, \Sigma, n, d,\left(d_{i j}\right)\right)$, and the corresponding $\tilde{n}$, to be fixed. We will also mostly assume that each partition $\left(d_{i j}\right)_{j=1, \ldots, m_{i}}$ is different from $(1, \ldots, 1)$, for in this case we could just reduce $n$.

Remark 1.1 A perhaps more traditional viewpoint is to consider only $\Sigma, n, d,\left(d_{i j}\right)$ to be given, and then determine the corresponding $\widetilde{\Sigma}$ using Conditions (1), (3), and (4). In this context, these conditions are replaced by the requirement that $\tilde{n}+d \cdot(\chi(\Sigma)-n)$ should be at most 2 . However, for non-orientable $\Sigma$ and even $d$, two possibilities exist for $\widetilde{\Sigma}$, so we prefer to stick to our datum which also includes $\widetilde{\Sigma}$. 
Even if we have stated the problem in full generality, we will confine ourselves in the rest of this section to the case $\Sigma=\mathbb{S}$, the sphere, because a full solution has been obtained (in the affirmative) whenever $\chi(\Sigma) \leqslant 0$, and the case $\Sigma=\mathbb{P}$, the projective plane, reduces to the case $\Sigma=\mathbb{S}$. Among the many sources for this solution, that we will discuss in Section 2, we single out here the fundamental contribution of Edmonds, Kulkarni and Stong [4], that we will frequently refer to, and the more recent one by Baránski [2]. We also mention the very interesting paper by Zheng [24], which introduces a new approach to the problem and describes many experimental results.

Motivation Surfaces are central objects in mathematics. They are interesting on their own (being the subject matter of, for instance, Teichmüller theory) and they are relevant to diverse fields such as algebraic geometry, complex analysis, and three-dimensional topology. Branched coverings between surfaces naturally occur within all these fields of investigation, so the basic Hurwitz existence problem stated above can be viewed in our opinion as one of the crucial ones in modern mathematics.

As discussed in Section 2, the problem has indeed attracted enormous attention over about a century, including that of outstanding mathematicians. The general solution of the problem for $\Sigma=\mathbb{S}$ is however still missing, which suggests that the problem is actually rather hard.

Besides being intrinsically interesting and very difficult, the existence problem for branched coverings with prescribed branch data naturally emerges in several contexts. For instance, it is relevant to the study of generating sets of surface groups. The reason is that a branched covering between surfaces naturally induces an orbifold-covering between 2-orbifolds (see Section 3 below), and coverings of the latter type correspond to subgroups of orbifold fundamental groups. Therefore existence of a branched covering matching a given datum is equivalent to existence in a certain Fuchsian group of a subgroup with given signature (Singerman [19]), which can be applied to deciding whether an arbitrary generating set of a surface group is Nielsen-equivalent to the standard generating set.

There are enumerative aspects of the Hurwitz problem which are not directly faced in the present paper but have obvious connections with our topic, and these aspects are relevant to the Gromov-Witten theory of algebraic curves. The Hurwitz number associated to a given datum is the number of equivalences classes of coverings realizing this datum and, as indicated in Okounkov and Pandharipande [17], the stationary Gromov-Witten invariant of a curve is equal to the sum of the Hurwitz numbers associated to certain branch data, determined through a specific correspondence between branch data and descendants in Gromov-Witten theory. 
Our own motivation for picking up the existence problem was to investigate the behaviour of Matveev's complexity [13] for 3-manifolds under finite covering. Conjectural formulae for complexity have been given by Martelli and Petronio [12] for Seifert 3-manifolds, ie, for circle fibrations over 2-orbifolds, an important class of 3-manifolds which has been classified for a long time (see eg Matveev and Fomenko [6]). It turns out that a finite covering between Seifert 3-manifolds induces a covering between the corresponding base 2 -orbifolds, and such a covering can be interpreted as a branched covering between surfaces, as already mentioned above. Therefore understanding branched coverings between surfaces can be viewed as a first necessary step towards the analysis of coverings between Seifert 3-manifolds and hence of Matveev's complexity under such coverings.

New results A branch datum will be called exceptional if it is compatible but not realizable by any branched covering. As already mentioned, for $\Sigma=\mathbb{S}$ exceptional data are known to exist. All examples discussed in the literature refer to the case where $d$ is non-prime, $n$ is 3 , and $\widetilde{\Sigma}$ is also equal to $\mathbb{S}$.

The main results obtained in the paper are listed below. They improve the understanding of exceptional data in that they place most of the exceptions occurring for non-prime $d \leqslant 22$ and $\widetilde{\Sigma}=\Sigma=\mathbb{S}$ (as described by Zheng in [24; 23]) within infinite series of exceptions, thus providing some sort of explanation for their emergence, and they show that such systematic exceptions occur also for $\widetilde{\Sigma} \neq \mathbb{S}$. Moreover, Theorems 1.4 and 1.5 below show that there are several exceptional series of data with $n>3$ branching points.

A result of rather different nature is given by Theorem 1.7, which provides a simple condition for realizability. More such conditions, based on a generalization of the results of Baránski [2], will be described in [18].

Proposition 1.2 Let $d \geqslant 8$ be even and consider compatible branch data of the form $\left(\widetilde{\Sigma}, \mathbb{S}, 3, d,(2, \ldots, 2),(5,3,2, \ldots, 2),\left(d_{3 j}\right)_{j=1, \ldots, m_{3}}\right)$.

- If $\widetilde{\Sigma}=\mathbb{T}$, the torus, whence $m_{3}=2$, the datum is realizable if and only if $\left(d_{31}, d_{32}\right) \neq(d / 2, d / 2)$;

- If $\widetilde{\Sigma}=\mathbb{S}$, whence $m_{3}=4$, the datum is realizable if and only if $\left(d_{3 j}\right)_{j=1, \ldots, 4}$ does not have the form $(k, k, d / 2-k, d / 2-k)$ for some $k>0$, or $(d / 2, d / 6, d / 6, d / 6)$ for $d$ a multiple of 6 .

Proposition 1.3 Let $\left(\mathbb{S}, \mathbb{S}, 3, d,\left(d_{i j}\right)\right)$ be a compatible branch datum with even $d$ and $\left(d_{1 j}\right)=(2, \ldots, 2)$. If $\left(d_{2 j}\right)=(3,3,2, \ldots, 2)$ or $\left(d_{2 j}\right)=(3,2, \ldots, 2,1)$ then the datum is realizable if and only if $d_{31} \neq d / 2$. 
Theorem 1.4 Suppose that $d$ and all $d_{i j}$ for $i=1,2$ are multiples of some $k$ with $1<k<d$. If the branch datum $\left(\mathbb{S}, \mathbb{S}, n, d,\left(d_{i j}\right)\right)$ is realizable then $d_{i j} \leqslant d / k$ for $i=3, \ldots, n$ and for all $j$.

Theorem 1.5 Suppose that $d$ and all $d_{i j}$ for $i=1,2$ are even. If the branch datum $\left(\mathbb{S}, \mathbb{S}, n, d,\left(d_{i j}\right)\right)$ is realizable then $\left(d_{i j}\right)$ refines the partition $(d / 2, d / 2)$ for $i=$ $3, \ldots, n$.

Corollary 1.6 Suppose that $d$ is a multiple of $2 k$ for some $k$ with $1<k<d / 2$, that all $d_{1 j}$ are multiples of $k$, and that all $d_{i j}$ for $i=2,3$ are even. If the branch datum $\left(\mathbb{S}, \mathbb{S}, n, d,\left(d_{i j}\right)\right)$ is realizable then $d_{i j} \leqslant d / k$ for $i=2,3$ and $d_{i j} \leqslant d / 2 k$ for $i=4, \ldots, n$ and for all $j$.

The last three criteria are especially expected to cover very many exceptional branch data. For example they imply that all the following series of data are exceptional:

$$
\begin{aligned}
& (\mathbb{S}, \mathbb{S}, d / k+1, d,(k, \ldots, k),(k, \ldots, k),(d / k+1,1, \ldots, 1), \\
& (2,1, \ldots, 1), \ldots,(2,1, \ldots, 1)), \quad k \mid d, \\
& (\mathbb{S}, \mathbb{S}, d / 2+1, d,(2, \ldots, 2),(2, \ldots, 2),(2, \ldots, 2), \\
& (2,1, \ldots, 1), \ldots,(2,1, \ldots, 1)), \quad d \equiv 2 \quad \bmod 4, \\
& (\mathbb{S}, \mathbb{S}, d / 2 k+2, d,(k, \ldots, k),(2, \ldots, 2),(2, \ldots, 2),(d / 2 k+1,1, \ldots, 1), \\
& (2,1, \ldots, 1), \ldots,(2,1, \ldots, 1)), \quad 2 k \mid d .
\end{aligned}
$$

We note that the exceptionality of the first of these series was already conjectured in general and proved for $d \leqslant 20$ by Zheng in [24, Conjecture 16].

Theorem 1.7 Let $\left(\widetilde{\Sigma}, \mathbb{S}, 3, d,\left(d_{i j}\right)\right)$ be a compatible branch datum. Let $p \geqslant 3$ be odd and suppose that all $d_{i j}$ are divisible by $p$. Then the datum is realizable.

Techniques Various equivalent ways of formulating the Hurwitz existence problem, and techniques to attack it, were developed over the time. We only mention here that the main classical tool, which goes back to Hurwitz himself, is a reformulation of the problem in terms of permutations. The main techniques we employ are as follows:

- Dessins d'enfants This is a classical notion due to Grothendieck, introduced within the study of algebraic maps between Riemann surfaces. This topic is tightly related to our existence problem, but dessins d'enfants were never employed directly before to attack the problem itself, and our adjustment of the notion proved rather fruitful, leading to Propositions 1.2 and 1.3, and to Theorem 1.4. 
- Decomposability The second tool we use is based on the idea of expressing a covering as a composition of two non-trivial ones, and the corresponding idea of finding certain "block decompositions" (first considered by Ritt) of permutations with given cycle structures. This idea leads to Theorem 1.5 and Corollary 1.6. It also allows us to establish the only realizability result of this paper, Theorem 1.7 .

Comments on the new results A very efficient algorithm to treat the existence problem was developed in [24] by Zheng, who also produced a vast collection of experimental data listing all the exceptional data up to degree 22 . The number of these exceptions is very large, and it appears rather hard to detect any sensible pattern in the list. However, we note that over a half of these exceptions fall in the domain of Theorem 1.5 and a smaller, but still noticeable, percentage is covered by Theorem 1.4.

We also notice that these two theorems cover all the exceptional data with $d \leqslant 17$ and $n \geqslant 5$. In these cases, the exceptions with even $d$ are almost always covered by Theorem 1.5, although a few are explained by Theorem 1.4. At the level of $d=18$ there are only two exceptional data with $n \geqslant 5$ which are not covered by these results, and a few more appear with $19 \leqslant d \leqslant 22$. To appreciate the power of these statements, in particular for large $n$, notice also that the total number of exceptional data in these degrees, even of those with $n \geqslant 5$, is in the hundreds.

Comments on the new techniques A detailed account on the established methods for facing the question of realizability of branch data will be given in Section 2, but we would like to mention here that all these methods have a chiefly algebraic flavour, except Baránski's recent one.

The use we make of dessins d'enfants to prove Propositions 1.2 and 1.3, and Theorem 1.4 provides the first application of such a notion to the Hurwitz existence problem. Besides the fact that the results are valuable on their own, we consider it rather interesting to have a transparent geometric explanation of non-existence for infinite series of coverings. In addition, the method appears to be generalizable to more infinite series.

Even if very simple, our idea of analyzing coverings which should be, if existent, compositions of other coverings proved rather fruitful and also allowed us to explain non-realizability of many data in purely geometric terms, as already stated above.

Organization of the paper In Section 2 we quickly review the results previously known on the Hurwitz existence problem, with the aim of helping the reader put our new contributions in the right perspective. In Section 3 we describe the main approaches historically taken to face the problem (and used in the rest of our paper), also trying to 
make the relations between them completely transparent. In Section 4 we develop the technique of dessins d'enfants, which allows us to establish Propositions 1.2 and 1.3 and Theorem 1.4. In Section 5 we apply the technique of decomposability of coverings to prove Theorems 1.5 and 1.7 and Corollary 1.6.

Acknowledgements We are grateful to Laurent Bartholdi for his instructions on how to use the software GAP to run computer experiments. We also thank Sergei Matveev, Laura Mazzoni, Alexander Mednykh, and Joan Porti for helpful conversations, and the referee of the first version of this paper for very useful suggestions.

The first-named author was supported by the INTAS YS fellowship 03-55-1423. The second-named author was supported by the INTAS project "CalcoMet-GT" 03-51-3663.

\section{Known results and techniques}

In this section we outline the main partial solutions of the Hurwitz existence problem which have been obtained over the time.

Known results for $\Sigma \neq \mathbb{S}$ We begin by reviewing several results whose overall content is that, to get a complete solution of the Hurwitz existence problem, it would be sufficient to settle the case where the base surface $\Sigma$ is the sphere $\mathbb{S}$. The first theorem we cite is attributed to Shephardson in Ezell [5, page 125] and explicitly proved in Husemoller [9, Theorem 4] and Edmonds, Kulkarni and Stong [4, Proposition 3.3]:

Theorem 2.1 A compatible branch datum with $\Sigma$ orientable and $\chi(\Sigma) \leqslant 0$ is realizable.

The next result is proved in [5, Theorem 3.4] and [4, Proposition 3.3]:

Theorem 2.2 A compatible branch datum with $\Sigma$ and $\widetilde{\Sigma}$ non-orientable and $\chi(\Sigma) \leqslant 0$ is realizable.

We then quote the following elementary fact, stated in [4, Proposition 2.7], and its consequence [4, Proposition 3.4]:

Proposition 2.3 A compatible branch datum with $\Sigma$ non-orientable and $\widetilde{\Sigma}$ orientable is realizable if and only if it is possible to decompose for all $i$ the partition $\left(d_{i j}\right)_{j=1, \ldots, m_{i}}$ of $d$ into partitions $\left(d_{i j}^{\prime}\right)_{j=1}^{m_{i}^{\prime}}$ and $\left(d_{i j}^{\prime \prime}\right)_{j=1}^{m_{i}^{\prime \prime}}$ of $d / 2$ in such a way that the branch datum

$$
\left(\widetilde{\Sigma}, \Sigma^{\prime}, 2 n, d / 2,\left(d_{i j}^{\prime}\right),\left(d_{i j}^{\prime \prime}\right)\right)
$$

is realizable, where $\Sigma^{\prime}$ is the orientable double covering of $\Sigma$. 
Corollary 2.4 A compatible branch datum with $\Sigma$ non-orientable, $\widetilde{\Sigma}$ orientable and $\chi(\Sigma) \leqslant 0$ is realizable.

The next result is due to Edmonds, Kulkarni and Stong [4, Theorem 5.1]. We recall that $\mathbb{P}$ is the projective plane.

Theorem 2.5 A compatible branch datum with $\Sigma=\mathbb{P}$ and non-orientable $\widetilde{\Sigma}$ is realizable.

These theorems imply that only the following instances of the Hurwitz existence problem remain open:

- $\Sigma=\mathbb{S}$

- $\Sigma=\mathbb{P}$ and $\widetilde{\Sigma}$ orientable.

However, Proposition 2.3 reduces the latter instance to the former one, to which we will therefore confine ourselves henceforth.

Known results for $\Sigma=\mathbb{S}$ When the base surface is the sphere $\mathbb{S}$, not every compatible branch datum is realizable. The easiest example is given in degree $d=4$ with $n=3$ branching points by the partitions $(3,1),(2,2),(2,2)$, which implies that $\widetilde{\Sigma}$ is $\checkmark$ too. A proof follows from Proposition 2.9 below, established in Section 4. In the rest of our paper the base surface $\Sigma$ will always be the sphere $\mathbb{S}$. Recall that a branch datum is exceptional if it is compatible but not realizable.

We will now review the main existence and non-existence theorems proved in the literature. However, we will not attempt to give a comprehensive list of the abstract statements. Instead, we will concentrate on the results which can be applied in a more direct fashion. The following was established in [4, Proposition 5.7].

Theorem 2.6 For all non-prime $d$ there exist exceptional branch data of degree $d$ with $n=3$ and $\widetilde{\Sigma}=\mathbb{S}$.

Turning to existence, the most general known result appears to be the following:

Theorem 2.7 A compatible branch datum is realizable if one of the partitions $\left(d_{i j}\right)$ is given by $(d)$ only.

Algebraic ${ }^{3} \mathcal{G}$ Geometric Topology, Volume 6 (2006) 
This fact was first stated by Thom [20, Theorem 1] for $\widetilde{\Sigma}$ also equal to $\mathbb{S}$, was reproved by Khovanskii and Zdravkovska [10, Theorem 2] and Baranski [2, Theorem 6] in the same context, and was generalized to arbitrary $\widetilde{\Sigma}$ in [4, Proposition 5.2]. A variation on this result is given in [4, Proposition 5.3], where the realizable branch data with one partition of the form $(d-1,1)$ are classified. In [4, page 775] a similar classification is announced for data with $n=3$ and one partition of the form $(m, 1, \ldots, 1)$.

Theorems 2.6 and 2.7, together with Proposition 2.9 below, are the main known results relevant to the case $n=3$, which we are most interested in. However, there are also some results relevant to the case where $n$ is "large" (usually compared to $d$ ). In this respect, a major contribution of Edmonds, Kulkarni and Stong is the following [4, Theorem 5.4]:

Theorem 2.8 A branch datum with $d \neq 4$ and $n \cdot d-\tilde{n} \geqslant 3(d-1)$ is realizable. The exceptional data with $d=4$ are precisely those with partitions $(2,2), \ldots,(2,2),(3,1)$.

Since we do not consider partitions of $d$ of the form $(1, \ldots, 1)$, one easily sees that $n \cdot d-\tilde{n} \geqslant n$, so a consequence of this result is that for fixed $d \neq 4$ the number of exceptional branch data of degree $d$ is finite [4, Corollary 4.4]. The next result is established in [4, Corollary 6.4]:

Proposition 2.9 For $\widetilde{\Sigma}=\mathbb{S}, n=3$ and even $d$ a branch datum with partitions $(x, d-x),(2, \ldots, 2),(2, \ldots, 2)$ is realizable if and only if $x=d / 2$.

The following results are due to Baránski [2, Proposition 10, Theorem 12, Corollary 15]. We note that the first one extends Theorem 2.7 in the special case where $\widetilde{\Sigma}=\mathbb{S}$, while the second one implies that for fixed $d$ the number of exceptional branch data of degree $d$ with $\widetilde{\Sigma}=\mathbb{S}$ is finite (which was already known after [4] for arbitrary $\widetilde{\Sigma}$ and $d \neq 4)$.

Proposition 2.10 A branch datum with $\widetilde{\Sigma}=\mathbb{S}$ is realizable if there exists $r$ such that $m_{1}+\ldots+m_{r}=(r-1) \cdot d+1$.

Proposition 2.11 A compatible branch datum with $\widetilde{\Sigma}=\mathbb{S}$ and $n \geqslant d$ is realizable.

Proposition 2.12 A compatible branch datum with $\widetilde{\Sigma}=\mathbb{S}, d_{i j} \leqslant 2$ for all $i, j$, and $m_{i} \geqslant d-\sqrt{d / 2}$ for all $i$ is realizable.

Considering Theorem 2.6 and the fact that all known exceptional branch data occur with non-prime degree $d$, one is naturally led to conjecture that for prime $d$ the Hurwitz existence problem always has a positive solution. It is claimed in [4, page 787] that establishing this conjecture in the special case $n=3$ would imply the general case. 
Established techniques and related known results The proofs of Theorems 2.1 and 2.2 are based on a reformulation of the main problem in terms of representations of the fundamental group of the $n$-punctured surface $\Sigma$ into the symmetric group $\mathfrak{S}_{d}$. This technique, already alluded to several times above, goes back to Hurwitz himself [8, Section I.1] and was later revisited and refined by Ezell [5, Theorem 2.1], Singerman [19, Theorem 1], where a more complicated situation (which includes cusped surfaces) is considered, and Husemoller [9, Theorems 3 and 5], where surfaces with boundary are also accepted. The permutation technique will be carefully reviewed and reinterpreted below in Section 3. It is however worth remarking here that the main algebraic results on permutations leading to Theorems 2.1 and 2.2 were also established in an abstract context in [3].

In the special case where $\Sigma=\mathbb{S}$ and $n=3$, the Hurwitz existence problem can be reinterpreted in terms of algebraic maps between algebraic curves. In this context Belyi's theorem [22, Proposition 3] could be viewed, in a sense, as a solution of the problem, but the necessary and sufficient condition for existence it gives is an abstract algebraic one which it does not seem to be possible to check in practice.

The results of Baránski stated above are based on a geometric criterion [2, Lemma 5] for the realizability of a branched covering of $\mathbb{S}$ with $\widetilde{\Sigma}$ also equal to $\mathbb{S}$.

To conclude, we quickly discuss the question (already mentioned above) of counting the number of (suitably defined) equivalence classes of branched coverings realizing a given branch datum. This counting problem in fact goes back to Hurwitz as well, for whom it was a primary motivation and who solved it in [8, pages 16, 22] for the case $n=2 d-2$ and all partitions of the form $(2,1, \ldots, 1)$, with $\widetilde{\Sigma}=\mathbb{S}$. Complete formulae for the general case were given by Mednykh [14, page 138], [15, Theorem C]. Of course, a branch datum is realizable if and only if the corresponding counting formula returns a positive value, so these formulae give an implicit solution to the Hurwitz existence problem. But again the actual computation appears to be hopeless. A variation of Mednykh's formulae, where some explicit computation is possible, was recently derived in [16] for special types of branched coverings.

Computational aspects The realizability of any specific branch datum can in principle be checked either using the formulae of Mednykh just mentioned, or via Hurwitz's reformulation in terms of permutations. However the former approach is too complicated to be practical. The latter one is suited for computer implementation, but it involves operating with conjugacy classes of permutations which already in the case $n=3$ and $d=11$ are too huge for the capacity of today's computers. A much more efficient method was recently developed by Zheng in [24], where the existence problem was expressed in terms of coefficients of certain generating functions. As already mentioned, 
the formulae also established by Zheng for calculating these coefficients allowed him to treat exceptional data up to degree 22 , see $[24 ; 23]$.

\section{Alternative viewpoints on the problem}

We start by describing equivalent formulations of the Hurwitz existence problem in terms of ordinary coverings of surfaces with boundary and in terms of 2-orbifolds. In some cases, one of these reformulations will be easier to handle than the original viewpoint of branched coverings.

Surfaces with boundary Let $\Sigma_{n}$ denote the surface obtained from $\Sigma$ by removing $n$ open discs with disjoint closures. A branched covering realizing a datum $\left(\widetilde{\Sigma}, \Sigma, n, d,\left(d_{i j}\right)_{i=1, \ldots, n}^{j=1, \ldots, m_{i}}\right)$ induces a genuine covering $\widetilde{\Sigma}_{\widetilde{n}} \rightarrow \Sigma_{n}$ in which the $i$-th component of $\partial \Sigma_{n}$ is covered by $m_{i}$ components of $\partial \widetilde{\Sigma}_{\tilde{n}}$, and the degrees of the restrictions to these components are $\left(d_{i j}\right)_{j=1, \ldots, m_{i}}$. Conversely, any such genuine covering $\widetilde{\Sigma}_{\widetilde{n}} \rightarrow \Sigma_{n}$ induces a realization of the branch datum. We will therefore consider also such a covering a realization of the datum.

2-orbifolds Let $\left(\Sigma ; p_{1}, \ldots, p_{n}\right)$ denote the (closed locally orientable) 2-orbifold with underlying surface $\Sigma$ and cone points of orders $p_{1}, \ldots, p_{n}$. Choosing $p_{i}$ to be divisible by $d_{i j}$ for $j=1, \ldots, m_{i}$, a branched covering realizing a datum $\left(\widetilde{\Sigma}, \Sigma, n, d,\left(d_{i j}\right)_{i=1, \ldots, n}^{j=1, \ldots, m_{i}}\right)$ induces an orbifold-covering [21]

$$
\left(\widetilde{\Sigma} ; \frac{p_{1}}{d_{11}}, \ldots, \frac{p_{1}}{d_{1 m_{1}}}, \ldots, \frac{p_{n}}{d_{n 1}}, \ldots, \frac{p_{n}}{d_{n m_{n}}}\right) \rightarrow\left(\Sigma ; p_{1}, \ldots, p_{n}\right) .
$$

And again, with details that we can safely leave to the reader, a covering between 2-orbifolds induces a branched covering between surfaces.

Covering from permutations We will denote by $\mathbb{S}, \mathbb{T}$ and $\mathbb{P}$ the sphere, the torus and the projective plane respectively, whence by $g \mathbb{\mathbb { V }}=\mathbb{\mathbb { N }} \ldots \# \mathbb{\mathbb { V }}$ ( $g$ times) and $g \mathbb{P}=$ $\mathbb{P} \# \ldots \# \mathbb{P}$ ( $g$ times) the orientable and non-orientable surfaces of genus $g \geqslant 1$. The following group presentations are well-known:

$$
\begin{aligned}
& \pi_{1}\left((g \mathbb{\mathbb { T }})_{n}\right)=\left\langle a_{1}, b_{1}, \ldots, a_{g}, b_{g}, e_{1}, \ldots, e_{n} \mid\left[a_{1}, b_{1}\right] \cdots\left[a_{g}, b_{g}\right] \cdot e_{1} \cdots e_{n}\right\rangle \\
& \pi_{1}\left((g \mathbb{P})_{n}\right)=\left\langle c_{1}, \ldots, c_{g}, e_{1}, \ldots, e_{n} \mid c_{1}^{2} \cdots c_{g}^{2} \cdot e_{1} \cdots e_{n}\right\rangle .
\end{aligned}
$$

In both cases the $e_{i}$ 's are represented by the boundary circles. In the orientable case $a_{k}, b_{k}$ is a meridian-longitude pair on the $k$-th copy of $\mathbb{T}$. In the non-orientable case $c_{k}$ is the only non-trivial loop on the $k$-th copy of $\mathbb{P}$. 
The first alternative viewpoint on the Hurwitz existence problem described above allows one to establish the following fact, originally due to Hurwitz, Husemoller, Ezell, and Singerman. We quickly review the geometric argument underlying the proof because we will need it below.

Theorem 3.1 A compatible branch datum $\left(\widetilde{\Sigma}, \Sigma, n, d,\left(d_{i j}\right)\right)$ is realizable if and only if there exists a representation $\theta: \pi_{1}\left(\Sigma_{n}\right) \rightarrow \mathfrak{S}_{d}$ such that:

(1) $\operatorname{Im}(\theta)$ acts transitively on $\{1, \ldots, d\}$;

(2) $\theta\left(e_{i}\right)$ has precisely $m_{i}$ cycles of lengths $d_{i 1}, \ldots, d_{i m_{i}}$;

(3) For non-orientable $\Sigma$ and orientable $\widetilde{\Sigma}$, each permutation $\theta\left(c_{k}\right)$ consists of cycles of even length only.

Proof Our proof only works for $n>0$, ie, it does not for genuine coverings. Recall first [6, Chapter 2] that $\Sigma_{n}$ can be obtained as shown in Figure 1 from a disc $\Delta$ by
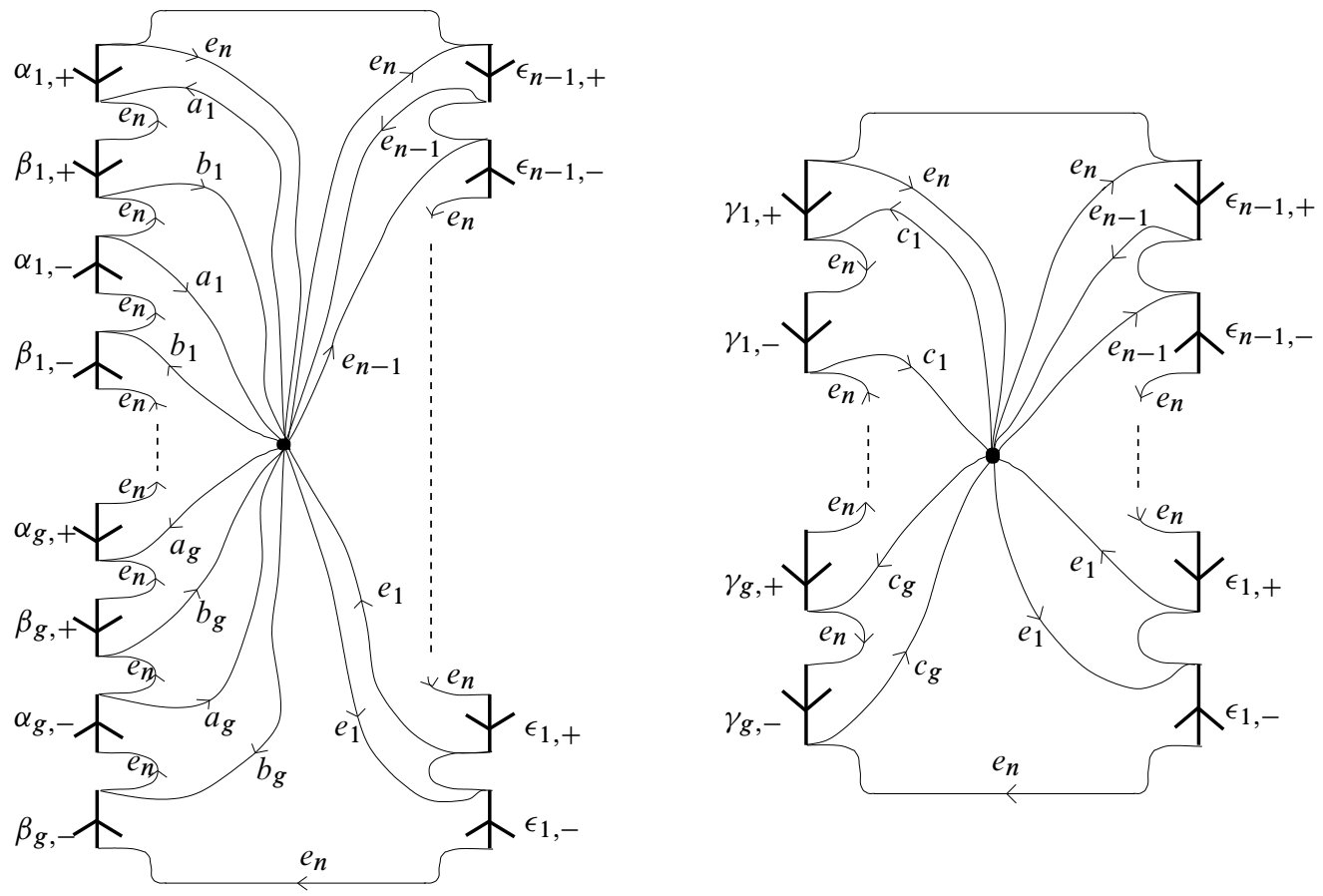

Figure 1: Construction of punctured surfaces

identifying each $\operatorname{arc} \epsilon_{i,-}$ to the $\operatorname{arc} \epsilon_{i,+}$ and, depending on orientability, either each 
$\alpha_{k,-}$ to $\alpha_{k,+}$ and $\beta_{k,-}$ to $\beta_{k,+}$, or each $\gamma_{k,-}$ to $\gamma_{k,+}$. The figure also shows loops corresponding to the generators of $\pi_{1}\left(\Sigma_{n}\right)$ used in the above presentations.

The degree- $d$ coverings of $\Sigma_{n}$ are now obtained as follows. We first take the disjoint union of $d$ copies $\left(\Delta^{(h)}\right)_{h=1}^{d}$ of $\Delta$, with the corresponding $\operatorname{arcs} \epsilon_{i, \pm}^{(h)}$ etc. Then we glue each $\epsilon_{i,-}^{(h)}$ to some $\epsilon_{i,+}^{\left(\theta\left(e_{i}\right)(h)\right)}$, where $\theta\left(e_{i}\right) \in \mathfrak{S}_{d}$, and similarly for the other arcs, using permutations $\theta\left(a_{k}\right)$ and $\theta\left(b_{k}\right)$, or $\theta\left(c_{k}\right)$. The corresponding covering is induced by the identification of each $\Delta^{(h)}$ with $\Delta$, and of course the covering is connected if and only if the subgroup of $\mathfrak{S}_{d}$ generated by $\theta\left(e_{1}\right), \ldots, \theta\left(e_{n-1}\right)$ and either the $\theta\left(a_{k}\right)$ 's and $\theta\left(b_{k}\right)$ 's or the $\theta\left(c_{k}\right)$ 's acts transitively on $\{1, \ldots, d\}$. It is also obvious that for $i=1, \ldots, n-1$ the way the $i$-th component of $\partial \Sigma_{n}$ is covered depends on the cyclic structure of $\theta\left(e_{i}\right)$ as described in the statement.

Considering the form of the presentation of $\pi_{1}\left(\Sigma_{n}\right)$ given above, we see that we can define $\theta\left(e_{n}\right) \in \mathfrak{S}_{d}$ in a unique fashion so to get a representation $\theta$. To conclude we must show that the way the $n$-th component of $\partial \Sigma_{n}$ is covered depends on the cyclic structure of $\theta\left(e_{n}\right)$, and that the covering over non-orientable $\Sigma_{n}$ is orientable if and only if each $\theta\left(c_{k}\right)$ contains cycles of even length only. Both assertions are easy and left as an exercise.

The parity condition Using Theorem 3.1 we can now explain Condition (2) of the definition of compatible branch datum.

Lemma 3.2 If a datum is realizable then $n \cdot d-\tilde{n}$ is even.

Proof The conclusion is evident when $\Sigma$ is orientable, so we assume it is not. Consider a representation $\theta$ realizing the datum as in Theorem 3.1. Notice that $\theta\left(e_{1}\right) \cdots \theta\left(e_{n}\right)$ is an even permutation, since its inverse is the product of either commutators or squares. A permutation is even if and only if it contains an even number of cycles of even length, ie, if the sum of a contribution $\ell-1$ for each cycle of length $\ell$ is even. Now the lengths of the cycles of $\theta\left(e_{i}\right)$ are the $d_{i j}$ 's, whence

$$
\sum_{i=1}^{n} \sum_{j=1}^{m_{i}}\left(d_{i j}-1\right)=n \cdot d-\tilde{n}
$$

is even.

\section{Exceptions via dessins d'enfants}

As mentioned in Section 1 many exceptional branch data exist when $\Sigma$ is the sphere $\mathbb{S}$, and in this section we present several classes of them, using a variation on Grothendieck's 
dessins d'enfants [7]. This notion in its original form is only relevant to the case of $n=3$ branching points, but we actually generalize it to arbitrary $n \geqslant 3$.

Definition 4.1 A dessin d'enfant on $\widetilde{\Sigma}$ is a graph $D \subset \widetilde{\Sigma}$ where:

(1) For some $n \geqslant 3$ the set of vertices of $D$ is split as $V_{1} \sqcup \ldots \sqcup V_{n-1}$ and the set of edges of $D$ is split as $E_{1} \sqcup \ldots \sqcup E_{n-2}$;

(2) For $i=1, \ldots, n-2$ each edge in $E_{i}$ joins a vertex of $V_{i}$ to one of $V_{i+1}$;

(3) For $i=2, \ldots, n-2$ any vertex of $V_{i}$ has even valence and going around it we alternatively encounter edges from $E_{i-1}$ and edges from $E_{i}$;

(4) $\widetilde{\Sigma} \backslash D$ consists of open discs.

The length of one of the discs in $\widetilde{\Sigma} \backslash D$ is the number of edges of $D$ along which the boundary of the disc passes (with multiplicity).

Proposition 4.2 The realizations of a branch datum $\left(\widetilde{\Sigma}, \mathbb{S}, n, d,\left(d_{i j}\right)\right)$ correspond to the dessins d'enfants $D \subset \widetilde{\Sigma}$ with the set of vertices split as $V_{1} \sqcup V_{2} \sqcup \ldots \sqcup V_{n-1}$ such that for $i=1$ and $i=n-1$ the vertices in $V_{i}$ have valences $\left(d_{i j}\right)_{j=1, \ldots, m_{i}}$, for $i=2, \ldots, n-2$ the vertices in $V_{i}$ have valences $\left(2 d_{i j}\right)_{j=1, \ldots, m_{i}}$, and the discs in $\widetilde{\Sigma} \backslash D$ have lengths $\left(2(n-2) d_{n j}\right)_{j=1, \ldots, m_{n}}$.

Proof Suppose a realization $f: \widetilde{\Sigma} \rightarrow \mathbb{S}$ exists, let the branching points be $x_{1}, \ldots, x_{n}$, for $i=1, \ldots, n-2$ choose a simple arc $\alpha_{i}$ joining $x_{i}$ to $x_{i+1}$, suppose the $\alpha_{i}$ 's meet at their ends only and avoid $x_{n}$, and let $\alpha$ be their union. Then define $D$ as $f^{-1}(\alpha)$ and set $V_{i}=f^{-1}\left(x_{i}\right)$ and $E_{i}=f^{-1}\left(\alpha_{i}\right)$. To conclude that $D$ is a dessin d'enfant with valences and lengths as required, the only non-obvious facts concern the components of $\widetilde{\Sigma} \backslash D$. But $\mathbb{S} \backslash \alpha$ is an open disc, the restriction of $f$ to any component of $\widetilde{\Sigma} \backslash D$ is a covering onto this disc with a single branching point, and such a covering is always modelled on the covering $z \mapsto z^{k}$ of the open unit disc onto itself, so the components of $\widetilde{\Sigma} \backslash D$ are open discs. More precisely, there is one such disc for each element of $f^{-1}\left(x_{n}\right)$, and it is easy to see that the $j$-th one has length as required.

Reversing this construction is a routine matter left to the reader.

From dessins to permutations and back We recall that the conjugacy classes in $\mathfrak{S}_{d}$ are given precisely by the partitions of $d$, with the class of a permutation being the array of lengths of its cycles. The following is a consequence of Theorem 3.1, in which we emphasize the constructive nature of the theorem: 
Corollary 4.3 The realizations of a branch datum $\left(\widetilde{\Sigma}, \mathbb{S}, n, d,\left(d_{i j}\right)\right)$ correspond to the choices of $\tau_{i}=\theta\left(e_{i}\right)$ in $\mathfrak{S}_{d}$ for $i=1, \ldots, n-1$ such that $\left\langle\tau_{1}, \ldots, \tau_{n-1}\right\rangle$ is transitive and, setting $\tau_{n}=\tau_{n-1}^{-1} \cdots \tau_{1}^{-1}$, for $i=1, \ldots, n$ the conjugacy class of $\tau_{i}$ is given by $\left(d_{i j}\right)_{j=1, \ldots, m_{i}}$.

Combining Proposition 4.2 and Corollary 4.3 we deduce a correspondence between (suitable) dessins d'enfants in $\widetilde{\Sigma}$ and (suitable) choices of $\tau_{1}, \ldots, \tau_{n-1} \in \mathfrak{S}_{d}$. Since we will use it in the sequel, we spell out this correspondence explicitly in the next two propositions. Proofs are easy and hence omitted.

Proposition 4.4 Given a dessin d'enfant $D$, with notation as in the definition, corresponding to a realization of a branch datum $\left(\widetilde{\Sigma}, \mathbb{S}, n, d,\left(d_{i j}\right)\right)$, permutations $\tau_{1}, \ldots, \tau_{n-1}$ corresponding to the same realization are constructed as follows:

- Enumerate the edges of $E_{i}$ as $e_{i}^{(1)}, \ldots, e_{i}^{(d)}$, starting in an arbitrary fashion for $E_{1}$ and so that for $i \geqslant 2$ around each vertex of $V_{i}$ each edge $e_{i}^{(k)}$ is followed by the edge $e_{i-1}^{(k)}$ with the same number $k$;

- For $i \leqslant n-2$ and $k \in\{1, \ldots, d\}$ select the vertex of $V_{i}$ to which $e_{i}^{(k)}$ is incident and define $\tau_{i}(k)$ to be $h$ such that the next $e_{i}^{(*)}$ around the vertex is $e_{i}^{(h)}$.

- For $k \in\{1, \ldots, d\}$ select the vertex of $V_{n-1}$ to which $e_{n-2}^{(k)}$ is incident and define $\tau_{n-1}(k)$ to be $h$ such that the next $e_{n-2}^{(*)}$ around the vertex is $e_{n-2}^{(h)}$.

Concerning this statement, recall that $\widetilde{\Sigma}$ is oriented and note the following about the edge $e_{i}^{(h)}$ needed to define $\tau_{i}(k)$ : for $i=1$ this edge comes immediately after $e_{i}^{(k)}$, while for $i=2, \ldots, n-2$ there is the edge $e_{i-1}^{(k)}$ located in between. Notice also that the edge $e_{n-2}^{(h)}$ when used in the definition of $\tau_{n-1}(k)$ again comes immediately after $e_{n-2}^{(k)}$.

To describe the opposite correspondence, given $\tau_{1}, \ldots, \tau_{n-1} \in \mathfrak{S}_{d}$, we construct a graph $D\left(\tau_{1}, \ldots, \tau_{n-1}\right)$ with vertices $V_{1} \sqcup \ldots \sqcup V_{n-1}$, where $V_{i}$ is the set of cycles of $\tau_{i}$, and for $i=1, \ldots, n-2$ and $k=1, \ldots, d$ an edge $e_{i}^{(k)}$ joins the cycles of $\tau_{i}$ and $\tau_{i+1}$ which contain $k$. Note that $D\left(\tau_{1}, \ldots, \tau_{n-1}\right)$ is connected if and only if $\left\langle\tau_{1}, \ldots, \tau_{n-1}\right\rangle$ is transitive. Then we consider in $D\left(\tau_{1}, \ldots, \tau_{n-1}\right)$ the loops constructed as follows: we start with some vertex of $V_{1}$ and follow the path $e_{1}^{(k)}, \ldots, e_{n-2}^{(k)}$. Having thus arrived to a vertex of $V_{n-1}$, we follow the path

$$
e_{n-2}^{\left(\tau_{n-1}^{-1}(k)\right)}, e_{n-3}^{\left(\tau_{n-2}^{-1} \tau_{n-1}^{-1}(k)\right)}, \ldots, e_{2}^{\left(\tau_{3}^{-1} \ldots \tau_{n-1}^{-1}(k)\right)}, e_{1}^{\left(\tau_{2}^{-1} \tau_{3}^{-1} \ldots \tau_{n-1}^{-1}(k)\right)}
$$

which takes us to a vertex of $V_{1}$. From there we proceed similarly starting from $e_{1}^{\left(\tau_{1}^{-1} \tau_{2}^{-1} \tau_{3}^{-1} \ldots \tau_{n-1}^{-1}(k)\right)}$ until we find the edge $e_{1}^{(k)}$ again. 
Proposition 4.5 Given $\tau_{1}, \ldots, \tau_{n-1} \in \mathfrak{S}_{d}$ corresponding to a realization of a branch datum $\left(\widetilde{\Sigma}, \mathbb{S}, n, d,\left(d_{i j}\right)\right)$, the space obtained from $D\left(\tau_{1}, \ldots, \tau_{n-1}\right)$ by attaching discs to the loops just described is $\widetilde{\Sigma}$, and $D\left(\tau_{1}, \ldots, \tau_{n-1}\right) \subset \widetilde{\Sigma}$ is a dessin d'enfant corresponding to the same realization of the branch datum.

A sample application To investigate the realizability of a given branch datum $\left(\widetilde{\Sigma}, \mathbb{S}, 3, d,\left(d_{i j}\right)\right)$ using the permutation approach of Corollary 4.3 , one should fix a certain $\tau_{1}$ with cycle lengths $\left(d_{1 j}\right)$ and then let $\tau_{2}$ vary in the conjugacy class $\left(d_{2 j}\right)$, checking that $\left\langle\tau_{1}, \tau_{2}\right\rangle$ is transitive and that $\tau_{1} \cdot \tau_{2}$ has cycle lengths $\left(d_{3 j}\right)$. Since conjugacy classes are huge, this method is only feasible for very small $d$, and we exploited it using the software GAP for $d \leqslant 10$ (but note that Zheng's alternative method [24] allows one to treat much higher degrees). On the other hand, the realizability criterion through dessins d'enfants, stated in Proposition 4.2, has the advantage of usually requiring the consideration of a much smaller number of cases. The geometric nature of the criterion often also makes it very easy to apply it. As a first example, we give a very simple proof of a result stated in Section 2 and originally established in [4].

Proof of Proposition 2.9 If the datum is realizable then the dessin d'enfant associated to the last two branching points is just a circle embedded in $\mathbb{S}$. Such a dessin decomposes $\mathbb{S}$ into two discs of the same length, so $x=d-x$, whence $x=d / 2$. The same argument proves also the opposite implication.

Graph fattening and applications To apply Proposition 4.2 it is sometimes useful to switch the viewpoint: instead of trying to embed a dessin $D$ in the surface $\widetilde{\Sigma}$, we try to thicken a given graph $D$ to a surface with boundary so to get $\widetilde{\Sigma}$ by capping off the boundary circles. An application of this method is given by the following proof of one of the results stated in Section 1.

Proof of Proposition 1.2 We give a unified proof. A dessin d'enfant $D$ corresponding to the first two partitions in the given branch datum, as an abstract graph, is homeomorphic to one of the graphs $X$ and $Y$ shown in Figure 2, with the two visible vertices
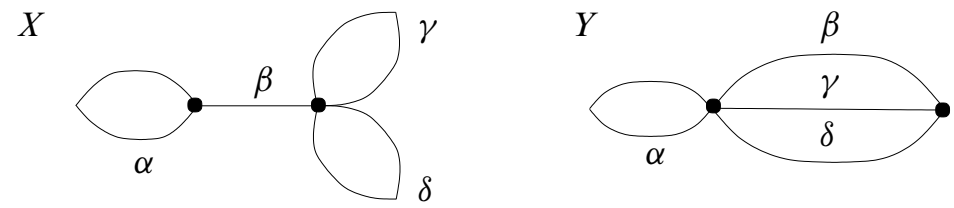

Figure 2: The two graphs with vertices of valences 5 and 3 
lying in $V_{2}$. To get $D$ we must then insert on each edge of $X$ or $Y$ an odd number of vertices belonging to $V_{1}$ and $V_{2}$ alternatively. With a slight abuse of notation, suppose we add $2 \alpha+1$ vertices on $\alpha$, then $2 \beta+1$ on $\beta$, and so on. Using the fact that $V_{1}$ has $d / 2$ vertices, we see that $\alpha+\beta+\gamma+\delta=d / 2-4$, and this is the only constraint on $\alpha, \beta, \gamma, \delta$.

Up to symmetry, the possible thickenings of $X$ and $Y$ to orientable surfaces with boundary are those described in Figure 3, as explained in the caption. The associated

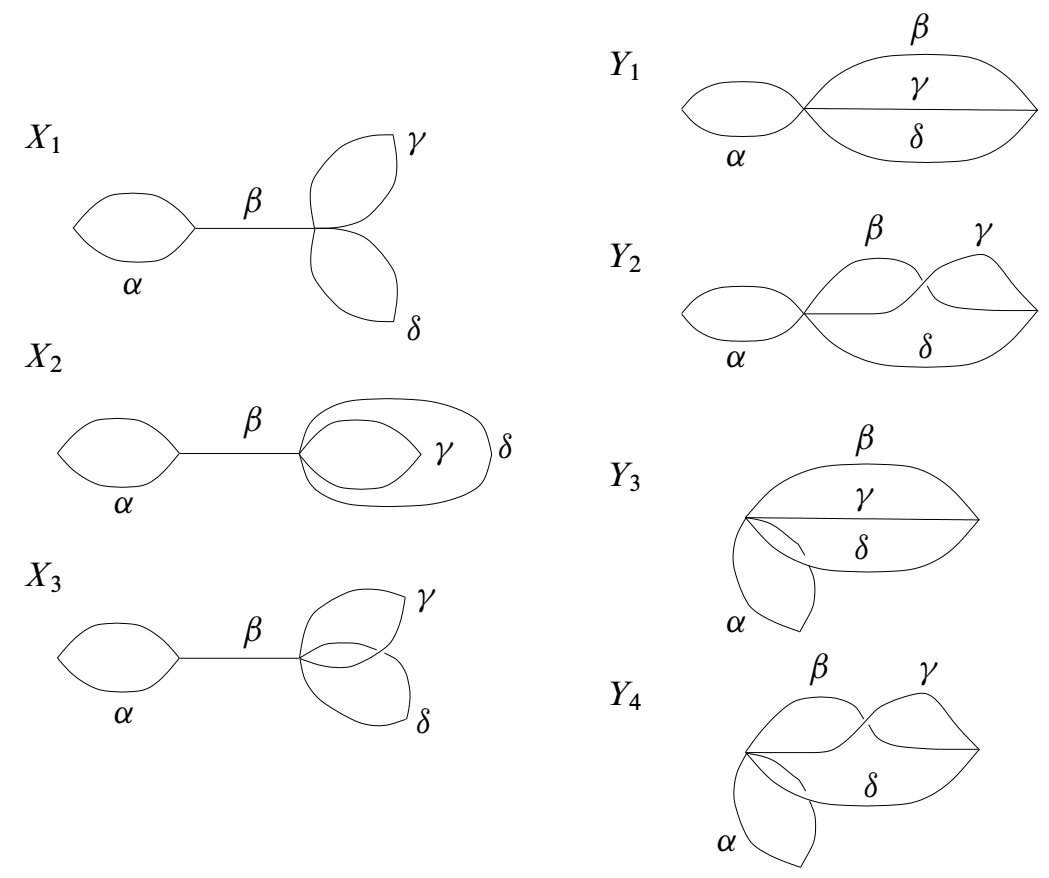

Figure 3: Thickenings of $X$ and $Y$, always given by the immersion in the plane

closed surfaces $\widetilde{\Sigma}$ and the half-lengths of the discs added are as follows:

$$
\begin{aligned}
& X_{1}: \mathbb{S},(\alpha+1, \gamma+1, \delta+1, \alpha+2 \beta+\gamma+\delta+5) \\
& X_{2}: \mathbb{S},(\alpha+1, \gamma+1, \gamma+\delta+2, \alpha+2 \beta+\delta+4) \\
& X_{3}: \mathbb{T},(\alpha+1, \alpha+2 \beta+2 \gamma+2 \delta+7) \\
& Y_{1}: \mathbb{S},(\alpha+1, \beta+\gamma+2, \gamma+\delta+2, \alpha+\beta+\delta+3) \\
& Y_{2}: \mathbb{T},(\alpha+1, \alpha+2 \beta+2 \gamma+2 \delta+7) \\
& Y_{3}: \mathbb{T},(\beta+\gamma+2,2 \alpha+\beta+\gamma+2 \delta+6) \\
& Y_{4}: \mathbb{T},(\alpha+\beta+\gamma+3, \alpha+\beta+\gamma+2 \delta+5) .
\end{aligned}
$$


We must then determine all the possible values which can be attained by these strings as $\alpha, \beta, \gamma, \delta$ vary among non-negative integers under the constraint $\alpha+\beta+\gamma+\delta=d / 2-4$.

Let us now specialize the proof for $\widetilde{\Sigma}=\mathbb{T}$, corresponding to cases $X_{3}, Y_{2}, Y_{3}$, and $Y_{4}$. It is obvious that the string $(d / 2, d / 2)$ cannot be realized. If $1 \leqslant k \leqslant d / 2-3$ we can realize $(d / 2+k, d / 2-k)$ using $Y_{4}$ with $\alpha=d / 2-3-k, \beta=\gamma=0, \delta=k-1$. Using $X_{3}$ and $Y_{3}$ we can obviously realize $(d-1,1)$ and $(d-2,2)$. This proves the first assertion.

Turning to $\widetilde{\Sigma}=\mathbb{S}$, let us first concentrate on case $Y_{1}$. We denote the unordered elements of $\left(d_{3 j}\right)_{j=1, \ldots, 4}$ by $x, y, z, w=d-x-y-z$, and solve in the unknowns $\alpha, \beta, \gamma, \delta$ the system

$$
\left\{\begin{array} { l } 
{ x = \alpha + 1 } \\
{ y = \beta + \gamma + 2 } \\
{ z = \gamma + \delta + 2 } \\
{ w = \alpha + \beta + \delta + 3 }
\end{array} \Rightarrow \left\{\begin{array}{l}
\alpha=x-1 \\
\beta=d / 2-x-z-1 \\
\gamma=x+y+z-1-d / 2 \\
\delta=d / 2-x-y-1
\end{array}\right.\right.
$$

This solution is acceptable if and only if $\alpha, \beta, \gamma, \delta$ are all non-negative, namely if the following holds:

$$
\text { (*) }\left\{\begin{aligned}
x+y & \leqslant d / 2-1 \\
x+z & \leqslant d / 2-1 \\
x+y+z & \geqslant d / 2+1 .
\end{aligned}\right.
$$

We deduce that a branch datum with $\widetilde{\Sigma}=\mathbb{S}$ is realizable using case $Y_{1}$ if and only if it is possible to extract from $\left(d_{3 j}\right)_{j=1, \ldots, 4}$ integers $x, y, z$ satisfying $(*)$. Let us now denote by $\ell$ the largest of the $d_{3 j}$ 's and prove the following facts:

Claim 1 If $\ell \geqslant d / 2$ then the branch datum cannot be realized using $Y_{1}$.

Claim 2 If $\ell<d / 2$ then the branch datum can be realized using $Y_{1}$ if and only if it does not have the form $(k, k, d / 2-k, d / 2-k)$.

Claim 1 is easy: of course we cannot choose $x, y$, or $z$ to be $\ell$, otherwise one of the first two conditions in $(*)$ would be violated, whence $x+y+z=d-\ell \leqslant d-d / 2=d / 2$, which contradicts the last condition in $(*)$. Turning to Claim 2 , it is clear that from $(k, k, d / 2-k, d / 2-k)$ we cannot extract $x, y, z$ satisfying $(*)$. To prove the converse, let us choose $x, y, z, w$ in increasing order, ie,

$$
x \leqslant y \leqslant z \leqslant w=\ell=d-x-y-z<d / 2
$$


which implies the last condition in $(*)$. Under these assumptions the second condition in $(*)$ implies the first one. If the second condition is violated then we have

$$
\begin{aligned}
& x \leqslant y \leqslant z=: d / 2-t \leqslant \ell=: d / 2-s \\
& x+z \geqslant d / 2 \Rightarrow x \geqslant t \\
& x+y=t+s \Rightarrow y \leqslant s \\
& z \leqslant \ell \Rightarrow t \geqslant s .
\end{aligned}
$$

These facts imply that $x \leqslant y \leqslant s \leqslant t \leqslant x$. Calling $k$ this common value, we deduce that $(x, y, z, w)$ has the form $(k, k, d / 2-k, d / 2-k)$, and the claim is established.

To conclude the proof for $\widetilde{\Sigma}=\mathbb{S}$ it is now sufficient to establish the following:

Claim 3 If $\ell \geqslant d / 2$ then a branch datum can be realized using $X_{1}$ or $X_{2}$ if and only if $\left(d_{3 j}\right)_{j=1, \ldots, 4}$ is not $(d / 2, d / 6, d / 6, d / 6)$.

Claim 4 No branch datum with $\left(d_{3 j}\right)_{j=1, \ldots, 4}$ of the form $(k, k, d / 2-k, d / 2-k)$ can be realized using $X_{1}$ or $X_{2}$.

Let us prove Claim 3. Again we denote the $d_{3 j}$ 's by $x, y, z, w$, and we choose them in increasing order, ie,

$$
x \leqslant y \leqslant z<w=\ell=d-x-y-z,
$$

whence $x+y+z \leqslant d / 2$. If we want to realize the datum using $X_{1}$ we have the following forced choice up to symmetry:

$$
\left\{\begin{array} { l } 
{ x = \alpha + 1 } \\
{ y = \gamma + 1 } \\
{ z = \delta + 1 } \\
{ w = \alpha + 2 \beta + \gamma + \delta + 5 }
\end{array} \quad \Rightarrow \quad \left\{\begin{array}{l}
\alpha=x-1 \\
\beta=d / 2-x-y-z-1 \\
\gamma=y-1 \\
\delta=z-1 .
\end{array}\right.\right.
$$

This is an acceptable solution unless $x+y+z=d / 2$. So we turn to case $X_{2}$ and try to realize the case $x \leqslant y \leqslant z<\ell=x+y+z$. We first solve

$$
\left\{\begin{array} { r l } 
{ x } & { = \alpha + 1 } \\
{ y } & { = \gamma + 1 } \\
{ z } & { = \gamma + \delta + 2 } \\
{ x + y + z } & { = \alpha + 2 \beta + \delta + 4 }
\end{array} \quad \Rightarrow \quad \left\{\begin{array}{l}
\alpha=x-1 \\
\beta=y-1 \\
\gamma=y-1 \\
\delta=z-y-1
\end{array}\right.\right.
$$

Algebraic $8 \mathcal{G}$ Geometric Topology, Volume 6 (2006) 
which is acceptable only for $z>y$. We are left to deal with the case $x \leqslant y=z<\ell=$ $x+2 y$ and we try to use $X_{2}$ solving

$$
\left\{\begin{array} { r l } 
{ y } & { = \alpha + 1 } \\
{ x } & { = \gamma + 1 } \\
{ y } & { = \gamma + \delta + 2 } \\
{ x + 2 y } & { = \alpha + 2 \beta + \delta + 4 }
\end{array} \quad \Rightarrow \quad \left\{\begin{array}{l}
\alpha=y-1 \\
\beta=x-1 \\
\gamma=x-1 \\
\delta=y-x-1
\end{array}\right.\right.
$$

which is acceptable only for $y>x$. We have thus realized the branch datum for all $\left(d_{3 j}\right)_{j=1, \ldots, 4}$ except $(d / 2, d / 6, d / 6, d / 6)$, which of course cannot be realized.

Turning to Claim 4, we begin using $X_{2}$. Since $\gamma+1<\gamma+\delta+2$ and $\alpha+1<$ $\alpha+2 \beta+\delta+4$, there is only one attempt we can make:

$$
\left\{\begin{array} { r l } 
{ k } & { = \alpha + 1 } \\
{ k } & { = \gamma + 1 } \\
{ d / 2 - k } & { = \gamma + \delta + 2 } \\
{ d / 2 - k } & { = \alpha + 2 \beta + \delta + 4 }
\end{array} \quad \Rightarrow \quad \left\{\begin{array}{l}
\alpha=k-1 \\
\beta=-1 \\
\gamma=k-1 \\
\delta=d / 2-2 k-1
\end{array}\right.\right.
$$

which is not acceptable. We then try $X_{1}$. Up to symmetry there is again one case only, which is of course impossible.

A similar argument allows one to prove Proposition 1.3.

Exceptional data with non-prime degree Here we obtain the proof of Theorem 1.4. We start with a lemma.

Lemma 4.6 Suppose that $d=k h$ with $k, h \geqslant 2$. Let $\left(s_{j}\right)_{j=1, \ldots, p},\left(t_{j}\right)_{j=1, \ldots, q}$ be partitions of $h$ with $p, q \geqslant 2$ and $p+q \geqslant h+2$. Then for all $1 \leqslant r<(p+q-h)$, if $n=p+q-r-h+2$, the following branch datum is exceptional:

$$
\begin{array}{r}
\left(\mathbb{S}, \mathbb{S}, n, d,\left(k s_{1}, \ldots, k s_{p}\right),\left(k t_{1}, \ldots, k t_{q}\right),(h+r, 1, \ldots, 1),\right. \\
(2,1, \ldots, 1), \ldots,(2,1, \ldots, 1)) .
\end{array}
$$

Proof It is easy to see that the datum is compatible. The proof of exceptionality is by induction on $n$. The base of induction is $n=3$. We remark that in this case the statement of the lemma could be inferred from results announced by Edmonds, Kulkarni, and Stong [4, page 775], which are however stated without proof, so for the sake of completeness we provide an independent argument.

When $n=3$, the branch datum does not contain partitions $(2,1, \ldots, 1)$. To prove the lemma in this case, we proceed by induction on $h$. If $h=2$ we have $p=q=2$ and $\left(t_{1}, t_{2}\right)=\left(s_{1}, s_{2}\right)=(1,1)$, while for $h \geqslant 3$ we have two cases: either one of the 
partitions $\left(t_{j}\right)_{j=1, \ldots, p}$ and $\left(s_{j}\right)_{j=1, \ldots, q}$ has the form $(1, \ldots, 1)$ or not. So the next claim serves both to prove the base step of the induction and to deal with the first case of the inductive step.

Claim Suppose that $k, h \geqslant 2$. Let $\left(s_{j}\right)_{j=1, \ldots, p}$ be a partition of $h$ with $p \geqslant 2$. Then the branch datum $\left(\mathbb{S}, \mathbb{S}, 3, k h,\left(k s_{1}, \ldots, k s_{p}\right),(k, \ldots, k),(h+p-1,1, \ldots, 1)\right)$ is exceptional.

First note that the number of 1 's in the third partition is $(k-1) h-p+1$. Take arbitrary $\tau_{1}, \tau_{2} \in \mathfrak{S}_{k h}$ with cyclic structures $\left(k s_{1}, \ldots, k s_{p}\right)$ and $(k, \ldots, k)$ such that $\left\langle\tau_{1}, \tau_{2}\right\rangle$ is transitive, and consider the associated graph $D\left(\tau_{1}, \tau_{2}\right)$. If $\tau_{1}, \tau_{2}$ realize the above datum, $\tau_{1} \cdot \tau_{2}$ has $(k-1) h-p+1$ fixed points. Remark that to each fixed point of $\tau_{1} \cdot \tau_{2}$ there corresponds in $D\left(\tau_{1}, \tau_{2}\right)$ a pair of edges having the same ends. More precisely, let us define a multi-edge of $D\left(\tau_{1}, \tau_{2}\right)$ as the set of all edges having two given vertices as ends. Then one sees that a multi-edge $\varphi$ gives rise to at most \# $(\varphi)$ fixed points of $\tau_{1} \cdot \tau_{2}$, and all fixed points arise like this. The case of \# $(\varphi)$ fixed points actually occurs only if $\tau_{1}$ and $\tau_{2}$ contain cycles which are the inverse of each other, but this is easily recognized to be incompatible with the assumptions of the claim. So a multi-edge $\varphi$ contributes with at most \# $(\varphi)-1$ fixed points. We conclude that the total number of fixed points is at most the sum of all the multiplicities of the multi-edges, which is equal to $k h$, minus the number of multi-edges.

Let us now estimate the number of multi-edges. By definition of $D\left(\tau_{1}, \tau_{2}\right)$, the set of its vertices is split as $V_{1} \sqcup V_{2}$, where $V_{1}$ consists of $p$ vertices of valences $k s_{1}, \ldots, k s_{p}$ and $V_{2}$ consists of $h$ vertices of valence $k$. This implies that at least $s_{j}$ multi-edges are incident to the $j$-th vertex of $V_{1}$. However, if there are exactly $s_{j}$ multi-edges, connectedness of $D\left(\tau_{1}, \tau_{2}\right)$ easily implies that $p=1$, which was excluded. So there are at least $s_{j}+1$ multi-edges. Therefore there are in total at least $\sum_{j=1}^{p}\left(s_{j}+1\right)=h+p$ multi-edges. From the above we deduce that $\tau_{1} \cdot \tau_{2}$ has at most $k h-(h+p)=(k-1) h-p$ fixed points. This implies that $\tau_{1}, \tau_{2}$ cannot realize the branch datum, hence the Claim is established.

To conclude the inductive proof on $h$ (still with $n=3$, which is the base of our more general induction) we have to deal with the case where $h \geqslant 3$ and both partitions $\left(s_{j}\right)_{j=1, \ldots, p}$ and $\left(t_{j}\right)_{j=1, \ldots, q}$ contain at least one entry larger than 1 . This implies that $p, q \leqslant h-1$ and, taking into account the inequality $p+q \geqslant h+2$, that $p, q \geqslant 3$. Suppose that a datum as in the statement of the lemma (under the current assumptions) is realizable and consider the dessin d'enfant $D$ constructed as in the proof of Proposition 4.2. 
We first show that $D$ contains vertices $v$ and $u$ such that all the edges of $D$ incident to $v$ join $v$ to $u$. Indeed, notice that $\mathbb{S}^{2} \backslash D$ consists of some bigons and one $2(p+q-1)$ gon. Successively compressing each bigon into a single edge (see Figure 4), we get an

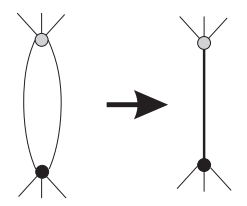

Figure 4: The passage from $D$ to $\bar{D}$

embedded graph $\bar{D}$ in $\mathbb{S}^{2}$ with the same vertices as $D$, whose complement consists of a single disc. Therefore $\bar{D}$ is a tree and not a point, so it contains vertices of valence 1 . Any such vertex $v$ and the vertex $u$ joined to it in $\bar{D}$ has the desired property.

Now let $D^{\prime}$ be the graph obtained from $D$ by deleting $v$ and all the edges incident to it. Obviously, $D^{\prime}$ is still a dessin d'enfant and its complement consists of some bigons and one $2(p+q-2)$-gon. Without loss of generality, we may assume that $v \in V_{2}$ and $u \in V_{1}$. If the valence of $v$ in $D$ is $k t_{a}$ and that of $u$ is $k s_{b}$ then the valence of $u$ in $D^{\prime}$ is $k\left(s_{b}-t_{a}\right)$ and this number is positive, otherwise $p=q=1$, which is excluded. Therefore $D^{\prime}$ realizes a branch datum as in the statement, with $n=3$, $k^{\prime}=k, h^{\prime}=h-t_{a}, p^{\prime}=p, q^{\prime}=q-1$, a partition $\left(s_{j}^{\prime}\right)$ of $h^{\prime}$ obtained from $\left(s_{j}\right)$ by replacing $s_{b}$ by $s_{b}-t_{a}$ and reordering, and another partition $\left(t_{j}^{\prime}\right)$ of $h^{\prime}$ obtained from $\left(t_{j}\right)$ by dropping $t_{a}$. To conclude the proof for the case $n=3$ we must show that the conditions $k^{\prime}, h^{\prime}, p^{\prime}, q^{\prime} \geqslant 2$ and $p^{\prime}+q^{\prime} \geqslant h^{\prime}+2$ are fulfilled. Of course $k^{\prime}, p^{\prime} \geqslant 2$. Moreover $q^{\prime} \geqslant 2$ because $q \geqslant 3$, which implies that $h^{\prime} \geqslant 2$. Now $h^{\prime}=h-t_{a} \leqslant h-1$ and $p+q \geqslant h+2$, so

$$
p^{\prime}+q^{\prime}=p+q-1 \geqslant h+2-1=h+1 \geqslant h^{\prime}+2 .
$$

The case $n=3$ is eventually settled.

Suppose now that $n \geqslant 4$, so the last partition has the form $(2,1, \ldots, 1)$. This implies that $p+q-h \geqslant r+2 \geqslant 3$, and, since $p, q \leqslant h$, we have $p, q \geqslant 3$. Assume, by contradiction, that the datum is realizable by a map $f$. Let $y_{i}$ be the branching point corresponding to the $i$-th partition and consider the dessin d'enfant $D$ constructed as in the proof of Proposition 4.2 with $x_{n-2}=y_{2}, x_{n-1}=y_{n}, x_{n}=y_{1}$ (the other $x_{j}$ 's must be the other $y_{j}$ 's, the order does not matter). In particular $D$ contains $f^{-1}\left(\alpha_{n-2} \cup \alpha_{n-3}\right)$, where $\alpha_{n-2}$ and $\alpha_{n-3}$ join $y_{n}$ to $y_{2}$ and $y_{2}$ to some other $y_{j}$ respectively. Recall that $V_{i}$ is $f^{-1}\left(x_{i}\right)$, so $V_{n-1}=f^{-1}\left(y_{n}\right)$ and $V_{n-2}=f^{-1}\left(y_{2}\right)$. Considering valences, one 
concludes that there are only two possibilities for $f^{-1}\left(\alpha_{n-2} \cup \alpha_{n-3}^{\prime}\right)$, where $\alpha_{n-3}^{\prime}$ is the half of $\alpha_{n-3}$ incident to $y_{2}$. These possibilities are shown in Figure 5, where the elements of $V_{n-1}$ are the black dots and those of $V_{n-2}$ are the grey dots. Let $e_{n-2}^{(i)}$,

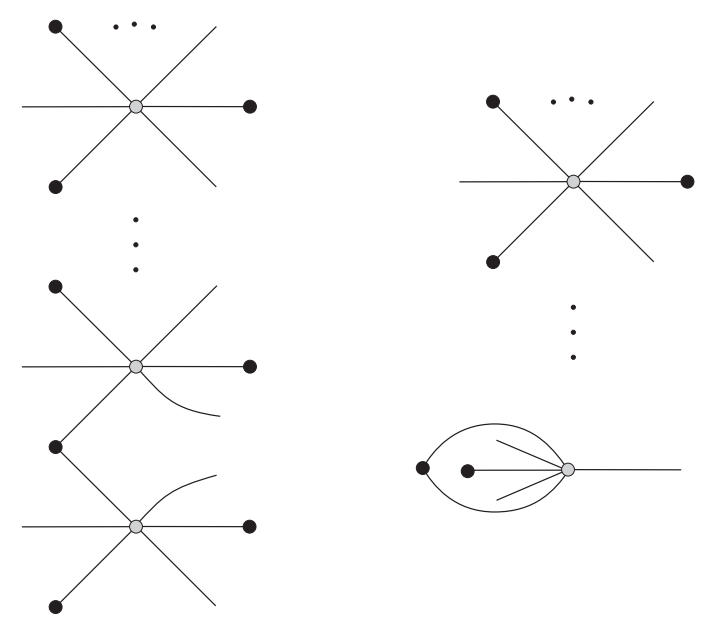

Figure 5: The two possibilities for $f^{-1}\left(\alpha_{n-2} \cup \alpha_{n-3}^{\prime}\right)$

$e_{n-2}^{(j)}$ be the edges with an endpoint at the 2-valent black vertex. Suppose we have the case shown in Figure 5-left, ie, that $e_{n-2}^{(i)}$ and $e_{n-2}^{(j)}$ are incident to two distinct grey vertices, which we denote by $v^{\prime}$ and $v^{\prime \prime}$. Then we remove all univalent black vertices together with the edge adjacent to any such vertex, and contract the set $e_{n-2}^{(i)} \cup e_{n-2}^{(j)}$ to a point, which now becomes a new grey vertex. This gives a new dessin d'enfant $D^{\prime}$ and we can now analyze which branch datum it realizes. We first notice that there is a natural correspondence between the complementary discs of $D^{\prime}$ and those of $D$, and the length of the $j$-th one of $D^{\prime}$ is $2 d_{1 j}=2 k s_{j}$ less than that of the $j$-th one of $D$. Moreover the contraction leading from $D$ to $D^{\prime}$ fuses $v^{\prime}$ and $v^{\prime \prime}$ together, so the number of grey vertices is decreased by one. Since exactly half of all the edges incident to a grey vertex belong to $E_{n-2}$, and all these edges are destroyed (either by removal or by contraction), the valence of any grey vertex distinct from $v^{\prime}, v^{\prime \prime}$ is halved, and the valence of the new vertex obtained by fusing is half the sum of the valences of $v^{\prime}$ and $v^{\prime \prime}$. However the grey vertices were non-terminal in $D$ but they become terminal in $D^{\prime}$. All this shows that $D^{\prime}$ is a dessin d'enfant realizing, up to re-ordering the $t_{j}$ 's and the entries in the second partition, the datum $\left(\mathbb{S}, \mathbb{S}, n-1, d,\left(k s_{1}, \ldots, k s_{p}\right),\left(k\left(t_{1}+\right.\right.\right.$ $\left.\left.\left.t_{2}\right), k t_{3}, \ldots, k t_{q}\right),(h+r, 1, \ldots, 1),(2,1, \ldots, 1), \ldots,(2,1, \ldots, 1)\right)$. Since $q \geqslant 3$ we again get a datum of the form described in the statement, but with $n-1$ in place of $n$. This contradicts the inductive assumption. 
If we have the case shown in Figure 5-right, we remove all the black vertices and all the edges incident to them. Then the valence of each grey vertex gets halved, and two of the complementary discs are merged into a single disc. A discussion similar to the above one shows that we get a dessin d'enfant realizing (up to reordering) the datum $\left(\mathbb{S}, \mathbb{S}, n-1, d,\left(k\left(s_{1}+s_{2}\right), k s_{3}, \ldots, k s_{p}\right),\left(k t_{1}, \ldots, k t_{q}\right),(h+\right.$ $r, 1, \ldots, 1),(2,1, \ldots, 1), \ldots,(2,1, \ldots, 1))$. As before, the datum is non-realizable by the inductive assumption, whence the conclusion in all cases.

Proof of Theorem 1.4 We assume $n \geqslant 3$ otherwise the statement is empty. We first claim that $m_{1}, m_{2} \geqslant 2$. By contradiction, suppose that $d_{11}=d$, all the $d_{2 j}$ 's are multiples of $k$, and $d_{31}>d / k$. Then $m_{1}=1, m_{2} \leqslant d / k, m_{3} \leqslant d-d / k$, and $m_{i} \leqslant d-1$ for $i \geqslant 4$. By the Riemann-Hurwitz condition $(n-2) d+2=$ $m_{1}+\ldots+m_{n} \leqslant 1+d / k+(d-d / k)+(n-3)(d-1)$, which implies that $n \leqslant 2$, a contradiction.

Suppose now that there is a realizable datum of the form

$\left(\mathbb{S}, \mathbb{S}, n, d,\left(k s_{1}, \ldots, k s_{p}\right),\left(k t_{1}, \ldots, k t_{q}\right),\left(d / k+r, d_{32}, \ldots, d_{3 m_{3}}\right),\left(d_{i j}\right)_{j=1, \ldots, m_{i}}\right)$,

with $r \geqslant 1$ and $p, q \geqslant 2$. By Theorem 3.1 we can find permutations $\tau_{1}, \ldots, \tau_{n}$ such that the cyclic structure of $\tau_{i}$ is given by the $i$-th partition and $\tau_{1} \tau_{2} \ldots \tau_{n}=1$. Obviously, we can present $\tau_{3}$ as a product of a cycle $\tau_{3}^{\prime}$ of length $d / k+r$ and a permutation $\tau_{3}^{\prime \prime}$ of cyclic structure $\left(1, \ldots, 1, d_{32}, \ldots, d_{3 m_{3}}\right)$. Notice that $\tau_{3}^{\prime \prime} \tau_{4} \ldots \tau_{n}$ can be obtained as the product of

$$
\left(d_{32}+\ldots+d_{3 m_{3}}-m_{3}+1\right)+\sum_{i=4}^{n}\left(d-m_{i}\right)=p+q-r-d / k-1
$$

transpositions. The collection of these transpositions together with the permutations $\tau_{1}, \tau_{2}, \tau_{3}^{\prime}$ provides thus a realization of the datum

$$
\begin{aligned}
& \left(\mathbb{S}, \mathbb{S}, p+q-r-d / k+2, d,\left(k s_{1}, \ldots, k s_{p}\right),\left(k t_{1}, \ldots, k t_{q}\right),(d / k+r, 1, \ldots, 1),\right. \\
& (2,1, \ldots, 1), \ldots,(2,1, \ldots, 1)) \text {, }
\end{aligned}
$$

which is non-realizable by Lemma 4.6, whence the conclusion.

\section{Exceptions due to decomposability}

We now describe another technique that can be employed to prove exceptionality of branch data. The basic underlying remark is that certain patterns in a branch datum force the covering realizing the datum to be decomposable, namely the composition of two non-trivial coverings, and one can deduce strong restrictions on the whole datum from the information that all its realizations are decomposable. 
Decomposability of coverings and permutations We will now state an easy result characterizing decomposable coverings, apparently due to Ritt and cited (in a less detailed fashion) in [11, Theorem 1.7.6]. We first introduce some terminology and establish a lemma which are necessary for the statement.

Definition 5.1 If $1<k<d$ and $k \mid d$ we call block decomposition of order $k$ for $\tau \in \mathfrak{S}_{d}$ a partition of $\{1,2, \ldots, d\}$ into $d / k$ subsets (the blocks) such that each block has $k$ elements and $\tau$ induces a well-defined permutation $\widehat{\tau}$ of the blocks. We say that $G<\mathfrak{S}_{d}$ has a block decomposition of order $k$ if there is a common such decomposition for all the elements of $G$.

Lemma 5.2 Let $\tau$ have cyclic structure $\left(d_{1}, \ldots, d_{m}\right)$. Then $\tau$ admits a block decomposition of order $k$ if and only if $\left(d_{1}, \ldots, d_{m}\right)$ can be partitioned into sets $D_{1}, \ldots, D_{t}$ and there exist integers $p_{1}, \ldots, p_{t} \geqslant 1$ such that for all $j$

$$
p_{j} \mid x \quad \forall x \in D_{j} \quad \text { and } \quad \sum_{x \in D_{j}} \frac{x}{p_{j}}=k .
$$

In this case $\widehat{\tau}$ has cyclic structure $\left(p_{1}, \ldots, p_{t}\right)$.

Proof Suppose the block decomposition exists, and take a cycle $\left(B_{1}, \ldots, B_{p}\right)$ of $\widehat{\tau}$, with $B_{j}=\left\{r_{j, 1}, \ldots, r_{j, k}\right\}$. Since $\tau\left(B_{j}\right)=B_{j+1}$ for $j=1, \ldots, p-1$, up to changing notation we have

$$
\tau\left(r_{1,1}\right)=r_{2,1} \quad \tau\left(r_{2,1}\right)=r_{3,1} \quad \ldots \quad \tau\left(r_{p-1,1}\right)=r_{p, 1} .
$$

Now we either have $\tau\left(r_{p, 1}\right)=r_{1,1}$ or, up to changing notation, $\tau\left(r_{p, 1}\right)=r_{1,2}$. Proceeding similarly we see that $\tau$ contains a cycle

$$
\left(r_{1,1}, \ldots, r_{p, 1}, r_{1,2}, \ldots, r_{p, 2}, \ldots, r_{1, b_{1}}, \ldots, r_{p, b_{1}}\right)
$$

of order $x_{1}=p \cdot b_{1}$. Repeating the same argument from $r_{1, b_{1}+1}$ and so on we find cycles of length $x_{1}, \ldots, x_{s}$ of $\tau$ such that $p \mid x_{l}$ and $\sum_{l=1}^{s} \frac{x_{l}}{p}=k$. The conclusion follows by considering the totality of the cycles of $\widehat{\tau}$, and the opposite implication is proved along the same lines.

Corollary 5.3 Let $\theta: \pi_{1}\left(\Sigma_{n}\right) \rightarrow \mathfrak{S}_{d}$ be associated as in Theorem 3.1 to a branched covering realizing a branch datum $\left(\widetilde{\Sigma}, \Sigma, n, d,\left(d_{i j}\right)\right)$. Then the covering is decomposable if and only if $\operatorname{Im}(\theta)$ has a block decomposition. Moreover, if $\operatorname{Im}(\theta)$ has a block decomposition of order $k$ with the decomposition of $\theta\left(e_{i}\right)$ corresponding to 
a partition of $\left(d_{i j}\right)$ into $D_{i 1}, \ldots, D_{i t_{i}}$ and integers $p_{i 1}, \ldots, p_{i t_{i}}$ then the covering factors through coverings realizing branch data

$$
\begin{gathered}
\left(\widetilde{\Sigma}, \Sigma^{\prime}, t_{1}+\ldots+t_{n}, k,\left(\left(\frac{x}{p_{i j}}\right)_{x \in D_{i j}}\right)_{i=1, \ldots, n, j=1, \ldots, t_{i}}\right) \\
\left(\Sigma^{\prime}, \Sigma, n, d / k,\left(\left(p_{i j}\right)_{j=1, \ldots, t_{i}}\right)_{i=1, \ldots, n}\right) .
\end{gathered}
$$

Note that to apply this result for any given $\theta$ one could use [1].

Very even data Here we apply the methods of the previous paragraph and those of Section 4 to describe two more infinite classes of exceptional data. Theorem 1.5 is in fact an immediate consequence of the following result:

Proposition 5.4 If $d$ is even and all $d_{i j}$ are also even for $i=1,2$ then any covering realizing a branch datum $\left(\mathbb{S}, \mathbb{S}, n, d,\left(d_{i j}\right)\right)$, up reordering the $d_{i j}$ 's, is a composition of coverings realizing data of the form

$$
\begin{aligned}
(\mathbb{S}, \mathbb{S}, 2 n-2, d / 2, & \left(d_{1 j} / 2\right)_{j=1, \ldots, m_{1}},\left(d_{2 j} / 2\right)_{j=1, \ldots, m_{2},}, \\
& \left(d_{3 j}\right)_{j=1, \ldots, h_{3}},\left(d_{3 j}\right)_{j=h_{3}+1, \ldots, m_{3}, \ldots,}, \\
& \left.\left(d_{n j}\right)_{j=1, \ldots, h_{n}},\left(d_{n j}\right)_{j=h_{n}+1, \ldots, m_{n}}\right),
\end{aligned}
$$

with $1 \leqslant h_{i}<m_{i}$ for $i=3, \ldots, n$, and

$$
(\mathbb{S}, \mathbb{S}, n, 2,(2),(2),(1,1), \ldots,(1,1)) .
$$

The proof requires a definition and an easy lemma. We call checkerboard graph a finite 1 -subcomplex of a surface whose complement consists of open discs each bearing a color black or white, so that each edge separates black from white.

Lemma 5.5 A connected graph in $\mathbb{S}$ with all vertices of even valence is a checkerboard graph.

Proof By induction on the number $v$ of vertices of the graph $D$. If $v=1$ then $D$ is a wedge of circles, and the conclusion follows from the fact that embedded circles are separating on $\mathbb{S}$. If $v>1$ choose an edge of $D$ having distinct ends, and let $D^{\prime}$ be obtained from $D$ by contracting this edge to a vertex. Then the hypothesis applies to $D^{\prime}$, so there is a checkerboard coloring of $\mathbb{S} \backslash D^{\prime}$. Now the regions of $\mathbb{S} \backslash D$ can be naturally identified to those of $\mathbb{S} \backslash D^{\prime}$, and it is easy to see that the coloring of $\mathbb{S} \backslash D^{\prime}$ also works for $\mathbb{S} \backslash D$. 
Proof of Proposition 5.4 Let $\tau_{1}, \ldots, \tau_{n-1} \in \mathfrak{S}_{d}$ correspond as in Corollary 4.3 to a realization of the given branch datum, with indices arranged so that $\tau_{1}$ and $\tau_{n-1}$ correspond to the first two partitions of $d$. We will prove that $\left\langle\tau_{1}, \ldots, \tau_{n-1}\right\rangle$ has a block decomposition of order $d / 2$ and then apply Corollary 5.3. Let $D=D\left(\tau_{1}, \ldots, \tau_{n-1}\right)$ be the dessin d'enfant associated to $\tau_{1}, \ldots, \tau_{n-1}$ as in Proposition 4.5. Then $D$ is a connected graph with all vertices of even valence, hence by Lemma 5.5 the components of $\mathbb{S} \backslash D$ have a checkerboard coloring.

We will now choose black and white colors also for the edges of $D$. We begin with the edges in $E_{1}$ and color them so that each $V_{1}$-corner of a black component of $\mathbb{S} \backslash D$ has first a black and then a white edge in the positive order around the vertex. Recalling that $\mathbb{S} \backslash D$ is checkerboard colored and that around each $v \in V_{2}$ the edges in $E_{1}$ and in $E_{2}$ alternate with each other, it is easy to see that all the edges in $E_{1}$ incident to $v$ have the same color. We then color the edges in $E_{2}$ incident to $v$ with the other color. Repeating this for all $v$ we color $E_{2}$, and we can proceed similarly to color all the edges of $D$.

Notice that by construction around any vertex of $D$ the colors of edges alternate, therefore for $i \geqslant 2$ the edges $e_{i-1}^{(k)}$ and $e_{i}^{(k)}$ always have opposite colors. Thus, if we define

$$
B_{i}=\left\{k: e_{i}^{(k)} \text { is black }\right\}, \quad W_{i}=\left\{k: e_{i}^{(k)} \text { is white }\right\}
$$

then we have $B_{1}=W_{2}=B_{3}=\ldots$ and $W_{1}=B_{2}=W_{3}=\ldots$. We denote the former set by $B$ and the latter set by $W$. The construction of $D$ given to prove Proposition 4.5 shows that $\tau_{2}, \ldots, \tau_{n-2}$ leave $B$ and $W$ invariant, while $\tau_{1}$ and $\tau_{n-1}$ switch them. Therefore we have a block decomposition of $\left\langle\tau_{1}, \ldots, \tau_{n-1}\right\rangle$ of order $d / 2$. Lemma 5.2 and Corollary 5.3 now imply that the covering $\widetilde{\Sigma} \rightarrow \mathbb{S}$ realizing the given branch datum factors through coverings $\widetilde{\Sigma} \rightarrow \Sigma^{\prime}$ and $\Sigma^{\prime} \rightarrow \Sigma$, with the latter having branch datum of the form $(\mathbb{S}, \mathbb{S}, n, 2,(2),(2),(1,1), \ldots,(1,1), *)$. But the only such covering is as in the statement and the conclusion easily follows.

Proof of Corollary 1.6 By Proposition 5.4 any covering realizing a datum as in the statement is a composition of the appropriate covering of degree 2 and a covering given by the datum

$$
\begin{aligned}
& \left(\mathbb{S}, \mathbb{S}, 2 n-2, d / 2,\left(d_{11}, \ldots, d_{1 h_{1}}\right),\left(d_{1\left(h_{1}+1\right)}, \ldots, d_{1 m_{1}}\right),\right. \\
& \left(d_{21} / 2, \ldots, d_{2 m_{2}} / 2\right),\left(d_{31} / 2, \ldots, d_{3 m_{3}} / 2\right) \text {, } \\
& \left(d_{4 j}\right)_{j=1, \ldots, h_{4}},\left(d_{4 j}\right)_{j=h_{4}+1, \ldots, m_{4}}, \ldots \text {, } \\
& \left.\left(d_{n j}\right)_{j=1, \ldots, h_{n}},\left(d_{n j}\right)_{j=h_{n}+1, \ldots, m_{n}}\right) \text {. }
\end{aligned}
$$

So by Theorem 1.4 we have $d_{i j} / 2 \leqslant d / 2 k$ for $i=2,3$ and $d_{i j} \leqslant d / 2 k$ for $i=4, \ldots, n$, whence the conclusion. 
We conclude the paper by establishing our only existence result.

Proof of Theorem 1.7 Let $\Sigma^{\prime}$ be the orientable surface with $\chi\left(\Sigma^{\prime}\right)=3-p$. Then the branch datum $\left(\Sigma^{\prime}, \mathbb{S}, 3, p,(p),(p),(p)\right)$ is compatible, whence realizable by Theorem 2.7. It is now easy to see that the datum $\left(\widetilde{\Sigma}, \Sigma^{\prime}, 3, d / p,\left(d_{i j} / p\right)\right)$ is also compatible. Now $\chi\left(\Sigma^{\prime}\right) \leqslant 0$, so the datum is realizable by Theorem 2.1, and the desired covering $\widetilde{\Sigma} \rightarrow \mathbb{S}$ can then be constructed as the composition of the coverings $\widetilde{\Sigma} \rightarrow \Sigma^{\prime}$ and $\Sigma^{\prime} \rightarrow \mathbb{S}$.

\section{References}

[1] MD Atkinson, An algorithm for finding the blocks of a permutation group, Math. Comput. 29 (1975) 911-913 MR0367030

[2] K Barański, On realizability of branched coverings of the sphere, Topology Appl. 116 (2001) 279-291 MR1857667

[3] G Boccara, Cycles comme produit de deux permutations de classes données, Discrete Math. 38 (1982) 129-142 MR676530

[4] A L Edmonds, R S Kulkarni, R E Stong, Realizability of branched coverings of surfaces, Trans. Amer. Math. Soc. 282 (1984) 773-790 MR732119

[5] C L Ezell, Branch point structure of covering maps onto nonorientable surfaces, Trans. Amer. Math. Soc. 243 (1978) 123-133 MR0500900

[6] A T Fomenko, S V Matveev, Algorithmic and computer methods for three-manifolds, Mathematics and its Applications 425, Kluwer Academic Publishers, Dordrecht (1997) MR1486574 Translated from the 1991 Russian original by M Tsaplina and M Hazewinkel and revised by the authors, with a preface by Hazewinkel

[7] A Grothendieck, Esquisse d'un programme (1984), from: "Geometric Galois actions. 1", (L Schneps, P Lochak, editors), London Mathematical Society Lecture Note Series 242, Cambridge University Press (1997) 5-48 MR1483106

[8] A Hurwitz, Ueber Riemann'sche Flächen mit gegebenen Verzweigungspunkten, Math. Ann. 39 (1891) 1-60 MR1510692

[9] D H Husemoller, Ramified coverings of Riemann surfaces, Duke Math. J. 29 (1962) 167-174 MR0136726

[10] A G Khovanskii, S Zdravkovska, Branched covers of $S^{2}$ and braid groups, J. Knot Theory Ramifications 5 (1996) 55-75 MR1373810

[11] S K Lando, A K Zvonkin, Graphs on surfaces and their applications, Encyclopaedia of Mathematical Sciences 141, Springer, Berlin (2004) MR2036721 With an appendix by D B Zagier 
[12] B Martelli, C Petronio, Complexity of geometric three-manifolds, Geom. Dedicata 108 (2004) 15-69 MR2112664

[13] S V Matveev, Complexity theory of three-dimensional manifolds, Acta Appl. Math. 19 (1990) 101-130 MR1074221

[14] A D Mednykh, Nonequivalent coverings of Riemann surfaces with a prescribed ramification type, Sibirsk. Mat. Zh. 25 (1984) 120-142 MR754748

[15] A D Mednykh, Branched coverings of Riemann surfaces whose branch orders coincide with the multiplicity, Comm. Algebra 18 (1990) 1517-1533 MR1059745

[16] S Monni, J S Song, Y S Song, The Hurwitz enumeration problem of branched covers and Hodge integrals, J. Geom. Phys. 50 (2004) 223-256 MR2078227

[17] A Okounkov, R Pandharipande, Gromov-Witten theory, Hurwitz theory, and completed cycles, Ann. of Math. (2) 163 (2006) 517-560 MR2199225

[18] E Pervova, C Petronio, On the existence of branched coverings between surfaces with prescribed branch data, II arXiv:math.GT/0611267

[19] D Singerman, Subgroups of Fuschian groups and finite permutation groups, Bull. London Math. Soc. 2 (1970) 319-323 MR0281805

[20] R Thom, L'équivalence d'une fonction différentiable et d'un polynôme, Topology 3 (1965) 297-307 MR0187249

[21] W P Thurston, Three-dimensional geometry and topology. Vol. 1, Edited by Silvio Levy, Princeton Mathematical Series 35, Princeton University Press, Princeton, NJ (1997) MR1435975

[22] J Wolfart, ABC for polynomials, dessins d'enfants, and uniformization - a survey, from: "Elementare und Analytische Zahlentheorie, (ELAZ May 24-28, 2004)", (W Schwarz, J Steuding, editors), Steiner Verlag, Stuttgart (2006) 313-345

[23] H Zheng, personal communication, May 2006

[24] H Zheng, Realizability of branched coverings of $\mathbb{S}^{2}$, to appear in Topol. Appl.

Chelyabinsk State University, ul. Br. Kashirinykh, 129

454021 Chelyabinsk, Russia

Dipartimento di Matematica Applicata, Università di Pisa

Largo Bruno Pontecorvo, 1, 56127 Pisa, Italy

pervova@csu.ru, petronio@dm.unipi.it

Received: 20 January 2006 Revised: 14 September 2006 